\title{
An important criterion for reliable multi-monochromatic x-ray imager diagnostics and its impact on the reconstructed images
}

\author{
T. Nagayama ${ }^{1}$, R.C. Mancini ${ }^{1}$, D. Mayes ${ }^{1}$, R. Tommasini ${ }^{2}$, and R. Florido ${ }^{3}$ \\ ${ }^{1}$ Physics Department, University of Nevada, Reno, NV 89557, USA \\ ${ }^{2}$ Lawrence Livermore National Laboratory, Livermore, CA 94550, USA \\ ${ }^{3}$ Departamento de Física, Universidad de Las Palmas de Gran Canaria, 35017 Las Palmas de Gran Canaria, Spain \\ (Received 10 April 2015; revised 13 June 2015; accepted 28 July 2015)
}

\begin{abstract}
Temperature and density asymmetry diagnosis is critical to advance inertial confinement fusion (ICF) science. A multimonochromatic x-ray imager, MMI, records the spectral signature from an ICF implosion core with time resolution, 2D spatial resolution and spectral resolution. While narrow-band images and 2D space-resolved spectra from the MMI data constrain the temperature and the density spatial structure of the core, the accuracy of the images and the spectra highly depends on the quality of the MMI data and the processing tools. Here, we synthetically investigate the criterion for reliable MMI diagnostics and its effects on the accuracy of the reconstructed images. The pinhole array tilt determines the object spatial sampling efficiency and the minimum reconstruction width, $w$. When the spectral width associated with $w$ is significantly narrower than the spectral linewidth, the line images reconstructed from the MMI data become reliable. The MMI setup has to be optimized for every application to meet this criterion for reliable ICF diagnostics.
\end{abstract}

Keywords: high power laser; inertial confinement fusion; monochromatic imager; $x$-ray diagnostics

\section{Introduction}

Inertial confinement fusion (ICF) is a concept for an alternative energy source, which releases energy by compressing a millimeter-scale capsule containing fusion fuel (i.e., hydrogen isotopes such as tritium, T, and deuterium, D), with ablation pressure driven by high power lasers ${ }^{[1,2]}$. Substantial progress has been made in theory, experiment and diagnostics of ICF, but efficient fuel burn, ignition, has not yet been achieved due to discrepancies between experiments and simulations ${ }^{[3]}$. While the sources of discrepancies have been extensively investigated and many of them have been recognized ${ }^{[4]}$, diagnostics that directly observe the evolution of the ICF implosion plasma spatial structure are desired to advance ICF science toward ignition.

Multi-monochromatic X-ray imagers (MMIs) are attractive instruments for this purpose. The MMI is a unique 2D spectrometer consisting of a pinhole array (PHA), a multilayered mirror (MLM) and a microchannel plate (MCP) ${ }^{[5,6]}$. It records an array of ICF implosion core images, each of which is formed by photons of slightly different wavelength.

Correspondence to: T. Nagayama, Sandia National Laboratories, Albuquerque, NM, USA. Email: tnnagay@sandia.gov
By processing MMI data, one can extract narrow-band images (i.e., intensity images of narrow spectral ranges), spaceintegrated spectra $^{[7-9]}$ and $2 \mathrm{D}$ space-resolved spectra ${ }^{[10]}$. Spectroscopic analysis of the images and spectra extracted from the MMI data can provide a variety of approaches to constrain the ICF plasma spatial structure in electron temperature, $T_{e}$, and electron density, $n_{e}$.

For example, we added a small amount of Ar into $\mathrm{D}_{2}$ fuel as a tracer and analyzed the Ar line emission to characterize the $T_{e}$ and $n_{e}$ of the core. Previously, we extracted timeresolved $2 \mathrm{D} T_{e}$ maps from two narrow-band images ${ }^{[11]}$. From a collection of space-resolved spectra, we extracted time-resolved 2D $T_{e}$ and $n_{e}$ maps $^{[10]}$. Synthetic investigations also suggest that time-resolved 3D $T_{e}$ and $n_{e}$ spatial distributions of ICF cores can be extracted by simultaneously analyzing collections of 2D space-resolved spectra extracted from three MMI instruments fielded along quasi-orthogonal lines of sight ${ }^{[12]}$. Thus, MMI diagnostics have the potential to better constrain ICF experiments and advance ICF theory.

While MMI data are rich in information, MMI data with good signal do not necessarily provide reliable information. To realize reliable MMI diagnostics, it is important to 
efficiently sample an object's spatial information. This requires the MMI setup to be optimized for every application.

In this paper, we discuss a criterion for reliable MMI diagnostics and show how this criterion affects the reliability of the extracted spatial information by reconstructing narrowband images from synthetic MMI data. It is difficult to experimentally study the fidelity of the reconstructed images because what is expected of the image is not known a priori. These questions can be best addressed using synthetic MMI data since the expected images can be computed. We show that the PHA tilt must be optimized to efficiently sample object spatial information and to minimize the minimum spectral width, $w$, required for the image reconstruction. This reconstruction width has to be significantly narrower than the full width at half maximum (FWHM) of the diagnostic lines.

This paper is organized as follows. Section 2 shows MMI data obtained with appropriate and inappropriate pinhole array designs and defines the criterion for reliable MMI diagnostics. Section 3 discusses the spectral model, which is used to create synthetic MMI data and also to compute expected images. In Section 4, the impact of the criterion is synthetically investigated using these models. The conclusions are given in Section 5.

\section{PHA design and image reconstruction}

The MMI consists of a hexagonally allocated PHA, a MLM and a MCP (Figure 1(a)), which provide 2D spatial resolution $(\Delta x \approx 11 \mu \mathrm{m})$, spectral resolution $(E / \Delta E \approx 150)$ and time resolution ( $\Delta t \approx 100 \mathrm{ps}$ ), respectively. The given resolutions are typical values for our applications ${ }^{[10-12]}$. The spatial resolution depends on pinhole size and object-PHAdetector distances. The spectral and temporal resolutions are determined by the specifications of the MLM and MCP, respectively. A key component for successful MMI data measurement is the PHA design, and it has to be optimized for every application. Here, we discuss necessary considerations to optimize the PHA design through the application to Ar-doped ICF experiments.

Pinhole size is determined based on the expected signal levels on the detector and the desired spatial resolution. The expected signal level on the detector depends on the object radiation power, the pinhole size, the object-PHA-detector distances and the detector sensitivity. For example, when the ICF implosion core is diagnosed with signals from $\mathrm{Ar}$ tracer, the core radiation power needs to be estimated with a spectral model at $T_{e}$ and $n_{e}$ predicted from the hydrodynamic simulations. If the estimated signals seem too low for a given configuration, one can increase the signals on the detector by increasing the object radiation power, increasing the object solid angle to the pinhole or improving the detector sensitivity. In our application, we field the instrument with a fixed PHA-detector distance as close as possible to

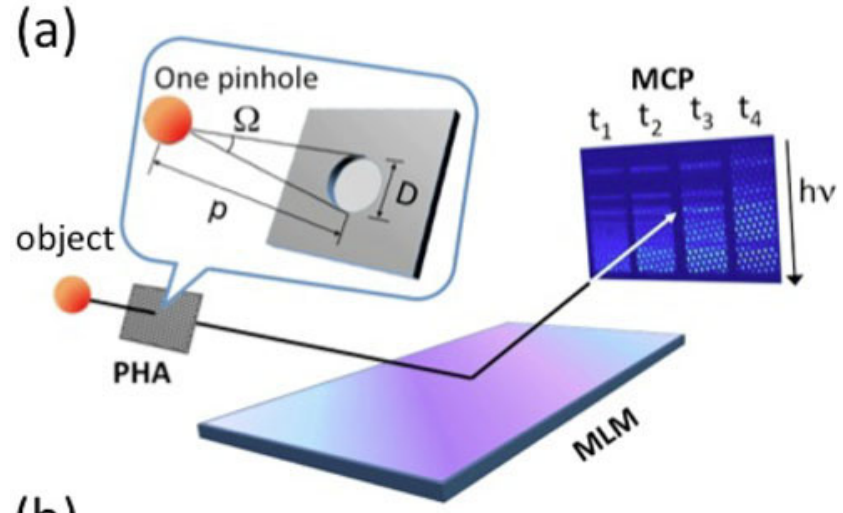

(b)
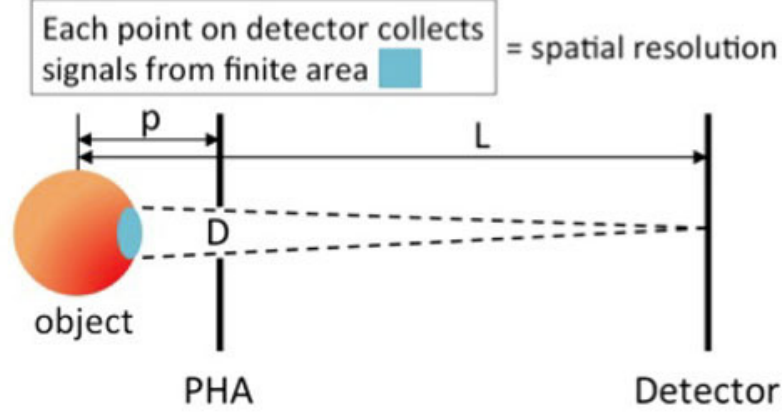

Spatial resolution $\approx D(1+1 / M)$

(c)

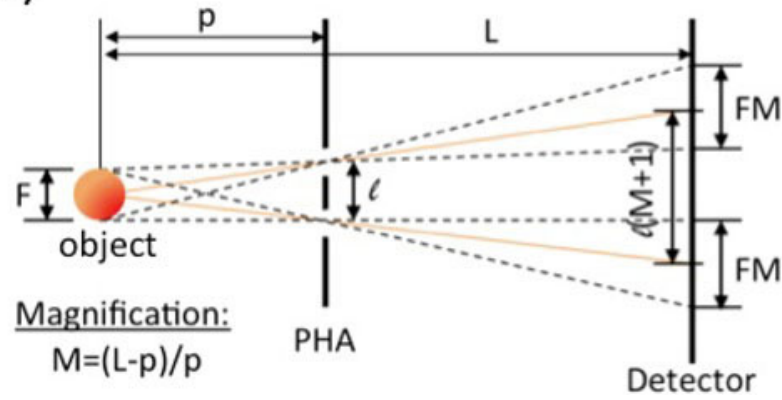

Figure 1. (a) The MMI consists of a PHA, a MLM and a MCP. The signal collected through a pinhole is proportional to the source-to-pinhole solid angle, $\Omega=\pi D^{2} / 4 p^{2}$. (b) The spatial resolution is determined by the object-pinhole-detector distances. (c) The magnification is determined by the object-pinhole distance, $p$, and the object-detector distance, $L$. The optimal pinhole separation, $l$, is determined so that images of size $F M$ do not overlap.

the object and use the x-ray framing camera available at OMEGA. This leaves us only two options for increasing the signal levels on the detector: (1) increasing the object solid angle to the pinhole by increasing the pinhole size (the blowup in Figure 1(a)) or (2) increasing the object radiation power by increasing the Ar tracer amount. One must consider the trade-off due to these options. For example, as the pinhole size is increased, the spatial resolution deteriorates because the signals observed at every point on the detector are integrated over a larger area on the source, as illustrated in Figure 1(b). When the Ar fraction is increased, one has to 
consider its effects on the implosion hydrodynamics ${ }^{[13]}$. The pinhole size needs to be optimized taking these details into account.

The pinholes are arranged in a hexagonal pattern because this allows the object images to fill the detector plane most efficiently. The pinhole separation, $l$, is optimized based on the target image size, $F M$, where $F$ is the object size and $M$ is the magnification (Figure 1(c)). The distance between adjacent image centers is $l(M+1)$, which defines the maximum image size allowed for a given $l$ and $M$. If the image size is larger than $l(M+1)$, the images start to overlap, which complicates the interpretation. Thus, $l$ is determined such that $F M \lesssim l(M+1)$.

Once the maximum allowed image size is defined, the appropriate tilt is determined based on the MCP strip width, $K$, so that the detector samples object spatial information most efficiently at each photon energy. In other words, the images aligned vertically across the MCP width should have a gradual horizontal shift so that, for every photon energy, they provide spatial information on a slightly different point in object space. The ideal tilt is computed by

$$
\theta=\arctan \left(\frac{\sqrt{4 / 3} l(M+1)}{K}\right)
$$

as discussed in Ref. [6].

An example of MMI data with an appropriate PHA tilt is shown in Figure 2(a). The object-to-pinhole distance, $p$, is $\approx 3 \mathrm{~cm}$; the object-to-detector distance, $L$, is $\approx 28.5 \mathrm{~cm}$ $(M \approx 8.5)$. The MLM consists of 300 bilayers of $\mathrm{W}$ and $\mathrm{BC}$, where the periodic thickness is $15 \AA$. Hexagonally arranged implosion core images are shown with a horizontal spectral axis. Due to K-shell line emission from $\mathrm{Ar}$ in the implosion core, images appearing around the photon energies associated with Ar Ly- $\alpha(\sim 3320 \mathrm{eV}), \mathrm{He}-\beta(\sim 3686 \mathrm{eV})$ and $\mathrm{Ly}-\beta(\sim 3935 \mathrm{eV})$ are brighter. The designed tilt angle based on Equation (1) was $8.95^{\circ}$ with respect to the vertical, and the data show $(8.66 \pm 0.10)^{\circ}$. As designed, this image array efficiently provides spatial information at each photon energy. For example, Figure 2(b) shows an image reconstructed from one pixel column of the MMI data at the He- $\beta$ line center (i.e., the orange line). Each pixel along this column is relocated into a single common image plane based on the position relative to its nearest image centers ${ }^{[9]}$. This single column provides strips of object spatial information that are uniformly separated over the image plane.

An example of MMI data with an inappropriate PHA tilt is shown in Figure 3(a). The MMI specification is identical to that of the previous example. While we used the same PHA design, it was misaligned in the MMI nose tip, which resulted in a significantly different tilt angle, $(5.22 \pm 0.02)^{\circ}$, in the data. The one pixel column image provides strips that

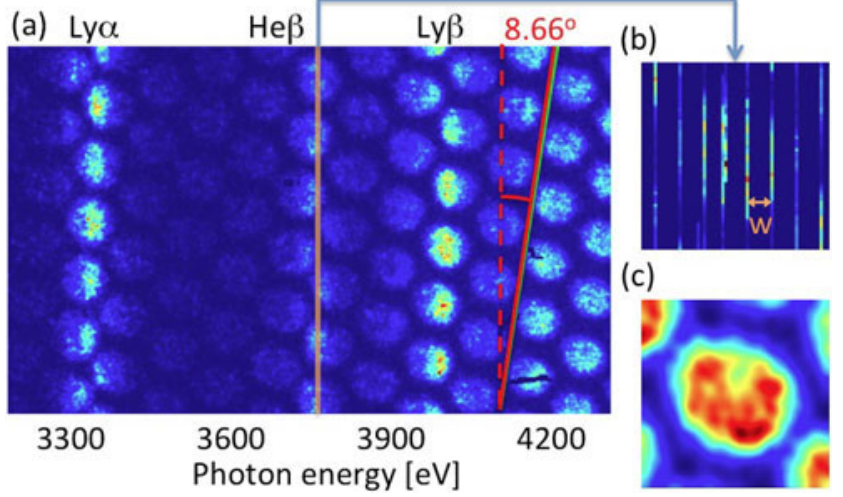

Figure 2. (a) MMI data with an appropriate PHA tilt. The dashed line is the reference vertical line (i.e., $\theta=0^{\circ}$ ). The red and green solid lines show the extracted $\left(8.66^{\circ}\right)$ and optimal $\left(8.95^{\circ}\right)$ PHA tilt angles. (b) Monochromatic image reconstructed from a single pixel column at the He- $\beta$ line center. (c) Narrow-band $\mathrm{He}-\beta$ image reconstructed over its linewidth (i.e., $\sim 60 \mathrm{eV}$ ).

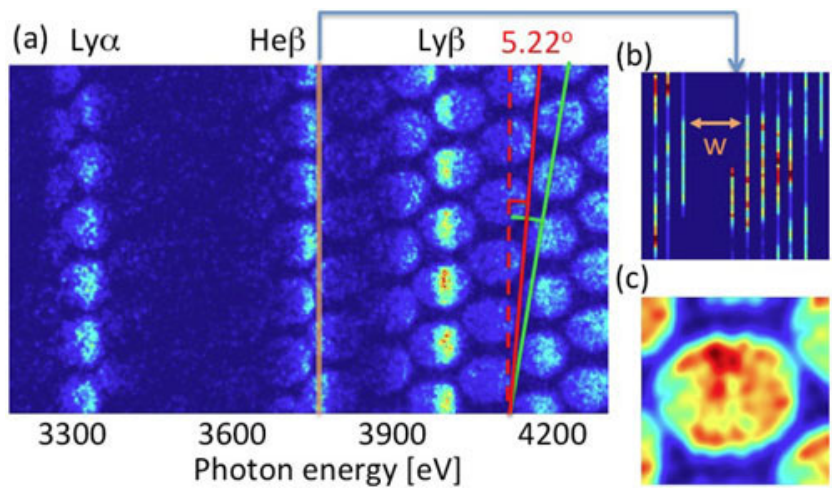

Figure 3. (a) MMI data with an inappropriate PHA tilt. The dashed line is the reference vertical line (i.e., $\theta=0^{\circ}$ ). The red and green solid lines show the extracted $\left(5.22^{\circ}\right)$ and optimal $\left(8.95^{\circ}\right)$ PHA tilt angles. (b) Monochromatic image reconstructed from a single pixel column at the He$\beta$ line center. (c) Narrow-band $\mathrm{He}-\beta$ image reconstructed over its linewidth (i.e., $\sim 60 \mathrm{eV}$ ).

are not uniformly distributed across the image, and thus the spatial sampling is biased (Figure 3(b)).

For both cases, one pixel column does not reconstruct the whole image, and adjacent pixel columns are needed which are at slightly different photon energies. Thus, the largest strip separation in the one pixel column image, $w$, defines the minimum width required to reconstruct the whole image, which we call the reconstruction width or sampling width. On one hand, the appropriate case shown in Figure 2 has $w=8$ pixels. Thus, eight pixel columns are needed to fully reconstruct the image, which correspond to $15 \mathrm{eV}$ for this particular dataset based on the extracted spectral axis ${ }^{[9]}$. Thus, the whole image can be reconstructed with a much narrower width than the spectral linewidth, where its FWHM is approximately $60 \mathrm{eV}$. By extending the narrow bandwidth to its FWHM, one can improve the signal-to-noise ratio $(\mathrm{S} / \mathrm{N})$ and form a reliable He- $\beta$ line image (Figure 2(c)). On the other hand, the inappropriate case shown in Figure 3 
has $w=20$ pixels, which corresponds to $44 \mathrm{eV}$. This is still narrower than the FWHM of the He- $\beta$ line. However, this means that the last pixel column added to form the whole image comes from $44 / 2=22 \mathrm{eV}$ away from the line center. The overall intensity from this pixel column is systematically lower than that of the line center, and the spatial information at the center of the gap, $w$, has to rely significantly on the intensity correction discussed in Equations (3) and (4) of Ref. [9]. Moreover, even after reconstructing the full image, the $\mathrm{S} / \mathrm{N}$ of the large gap region is still very low. Accordingly, the reconstructed image structure becomes less reliable around the large gap region.

To summarize, the PHA needs to be tilted at an appropriate angle to achieve efficient spatial sampling and minimize the reconstruction width, $w$. Reliable MMI diagnostics further require that the spectral width corresponding to $w$ is much narrower than the FWHM of the diagnostic lines such as $\mathrm{He}-\beta$ and Ly- $\beta$. Those linewidths need to be estimated from conditions predicted with hydrodynamic simulations and taken into account to optimize the PHA design. We note that, as long as the Stark linewidth is broader than the sampling width, the diagnostics should work. However, practically speaking, a Stark width of $60 \mathrm{eV}$ could still have spectral structures that change rapidly within the sampling width (e.g., structures around the center of the line). Thus, for reliable image reconstruction, $\Delta E_{\text {Stark }}>\Delta E_{\text {instrum }}>w$ is ideal, where $\Delta E_{\text {Stark }}$ and $\Delta E_{\text {instrum }}$ are the Stark width of the line and the spectral resolution of the instrument, respectively.

The extracted one pixel column image such as Figures 2(b) and 3(b) gives some idea of how reliable the recorded MMI data are. However, it does not directly answer how critical it is to satisfy this criterion. To better illustrate this point, we perform a synthetic investigation in Section 4 using the spectral model discussed in Section 3.

\section{Spectral model}

The spectral model used in this paper computes emergent spectra at every point on the image plane, $I_{v}(x, y)$, and then $I_{v}(x, y)$ is used to compute both synthetic MMI data and expected images. The case of investigation is an OMEGA direct-drive Ar-doped $\mathrm{D}_{2}$-filled ICF implosion because it has been one of our main applications of the MMI diagnostics $^{[10-12]}$. The ICF implosion core is the object for the synthetic data, and we define it as a sphere of uniform $T_{e}$ and $n_{e}$ to simplify the interpretation of the synthetic investigation. The capsule is initially filled with 20 atm of $\mathrm{D}_{2}$ with $0.18 \%$ atomic concentration of Ar. The Ar photonenergy-dependent emissivity and opacity are computed for given $T_{e}$ and $n_{e}$, and emergent spectra are computed by solving the radiation transport along each chord parallel to the line of sight $^{[12]}$.
To compute the Ar emissivity, $\epsilon_{\nu}$, and opacity, $\kappa_{\nu}$, necessary atomic data are computed with the Flexible Atomic Code $(\mathrm{FAC})^{[14]}$, and Ar level populations are solved for the given $T_{e}$ and $n_{e}$ with a collisional-radiative model, $\mathrm{ABAKO}^{[15]}$. We used the Stewart and Pyatt model ${ }^{[16]}$ for continuum lowering effects and the escape factor approximation to account for radiation transport effects on the populations ${ }^{[17]}$. The spectral emissivity and opacity are computed from the calculated populations including detailed Stark line profiles computed by MERL ${ }^{[18]}$.

Emergent spectra are computed along each chord on the image plane by numerically integrating the radiation transport equation assuming parallel ray tracing ${ }^{[19]}$ :

$$
\frac{d I_{v}(x, y)}{d z}=\epsilon_{v}(x, y, z)-I_{\nu}(x, y) \kappa_{v}(x, y, z)
$$

where the $z$ axis is parallel to the line of sight, the $x-y$ plane is parallel to the image plane of the detector, $I_{v}(x, y)$ is the emergent intensity at $(x, y)$ on the source $\left(\mathrm{erg} / \mathrm{s} / \mathrm{cm}^{2} / \mathrm{sr} / \mathrm{eV}\right.$ ) and $\tau_{v}$ is the optical depth from point $z$ in the object to the observer side of the object surface. The quantities $\epsilon_{v}(x, y, z)$ and $\kappa_{v}(x, y, z)$ are determined with ABAKO at the given $T_{e}$ and $n_{e}$ if $(x, y, z)$ is inside the object and zero if it is outside the object.

Potential inaccuracy in the calculated emissivity and opacity does not affect the conclusions derived from the synthetic investigations performed in this paper because $I_{v}(x, y)$ is used to calculate both the synthetic MMI data and the expected images. What we pursue is the discrepancies produced due to the discrete nature of MMI data and the details of data processing.

The expected narrow-band images are computed by integrating $I_{v}(x, y)$ over any given spectral range as

$$
I_{\Delta v}(x, y)=\int_{\Delta v} I_{\nu}(x, y) d v
$$

where $\Delta v$ is the bandwidth of interest.

Synthetic MMI data are computed from the emergent spectra, $I_{v}(x, y)$, assuming parallel ray tracing. When MMI data are processed as discussed in Ref. [9], each MMI pixel, $\left(x_{m m i}, y_{m m i}\right)$, uniquely defines its photon energy, $h v$, and its position on the image plane, $(x, y)$. Thus, using this pixel information provided from actual MMI data, one can compute each pixel of synthetic MMI data as $I_{v}(x, y) \Delta h v \Delta A$ by converting every pixel $\left(x_{m m i}, y_{m m i}\right)$ into $(x, y)$ and $h v$. Here, $\Delta h v$ and $\Delta A$ are the finite spectral width and the finite area at the pixel $\left(x_{m m i}, y_{m m i}\right)$.

One concern is the validity of parallel ray tracing for the synthetic MMI data modeling. While photons going through pinholes are not precisely parallel, the parallel ray assumption is still valid for our applications. Based on the 
object size of $100 \mu \mathrm{m}$ and object-PHA distance of $3 \mathrm{~cm}$, rays through a single pinhole deviate from parallel by less than $\theta<\arctan (100 \mu \mathrm{m} / 3 \mathrm{~cm})=0.2^{\circ}$. This maximum deviation in angle can be interpreted in terms of the maximum deviation on the image plane as $(100 \mu \mathrm{m}) \times \sin \left(0.2^{\circ}\right)<$ $0.3 \mu \mathrm{m}$, which is much smaller than the typical spatial resolution of the image, $\Delta x \approx 11 \mu \mathrm{m}$.

Photons through different pinholes have a larger deviation from the parallel rays. In typical data, pinholes used to project images on a single MCP are separated by a maximum of $\sim 2 \mathrm{~mm}$. Then, photons going through those pinholes could deviate from parallel by up to $\theta<$ $\arctan (2 \mathrm{~mm} / 3 \mathrm{~cm})=3.8^{\circ}$. Along the maximum chord length in the object, this results in a chord deviation of $(100 \mu \mathrm{m}) \times \sin \left(3.8^{\circ}\right)=6.6 \mu \mathrm{m}$ on the image plane. This deviation is still less than the spatial resolution of the instrument. Furthermore, this deviation is an upper bound because usually only a smaller portion of the MMI data is used in the analysis. For example, for $\mathrm{He}-\beta$ and $\mathrm{Ly}-\beta$ analysis, the most distant pinholes used in the analysis are separated by less than $1 \mathrm{~mm}$, and thus the deviation on the image plane is less than $3.3 \mu \mathrm{m}$. Hence, parallel ray tracing is a good approximation to interpret the photon paths through the object for many MMI applications, including ours.

\section{Impact of the criterion on MMI spatial sampling}

The quality of the MMI data depends on whether or not $w$ is significantly narrower than the spectral linewidth. In this section, we synthetically investigate the impact of the criterion on the reliability of the extracted spatial information. This can be demonstrated by reconstructing images from the synthetic MMI data that do and do not satisfy the criterion and comparing them with the expected images.

First, MMI data with an appropriate PHA are created using a uniform sphere of $100 \mu \mathrm{m}$ diameter, $T_{e}=1500$ and $n_{e}=1.5 \times 10^{24} \mathrm{~cm}^{-3}$. The PHA pattern and the photonenergy axis are those from the appropriate data shown in Figure 2(a). The conditions are the values representative of Figure 2(a) based on the analysis of its space-integrated spectra. Thus, as already discussed in Section $2, w=8$ pixels (i.e., $\sim 15 \mathrm{eV}$ ) and the FWHMs of the He- $\beta$ and Ly- $\beta$ lines are roughly $60 \mathrm{eV}$, which satisfies $w \ll$ FWHM.

Figure 4(a) shows the resultant synthetic MMI data. Because $w$ is much narrower than the FWHMs of the spectral lines, the reconstructed $\mathrm{He}-\beta$ and $\mathrm{Ly}-\beta$ images shown in Figure 4(b) agree very well with the expected images shown in Figure 4(c).

We note that there are small vertical structures introduced in the Ly- $\beta$ image in Figure 4(b). These structures come from the discrete nature of the MMI data. The MMI data have only a finite number of images, each of which spans slightly different photon-energy ranges. The processing tool combines the finite information from the different pinhole (a) Synthetic appropriate MMI data

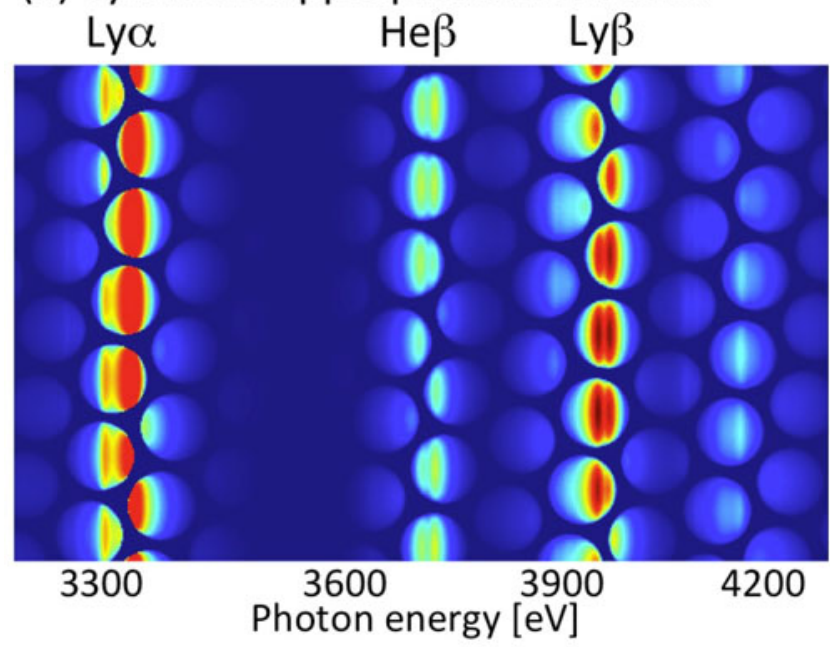

(b) Reconstructed images

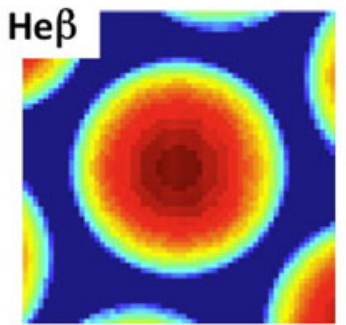

(c) Expected images

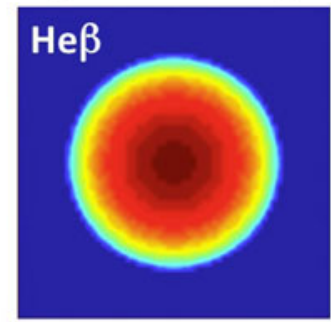

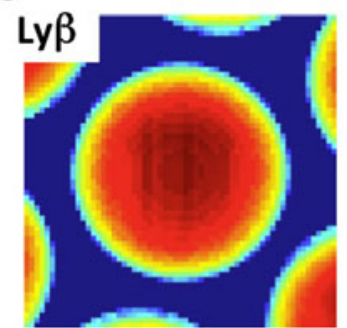

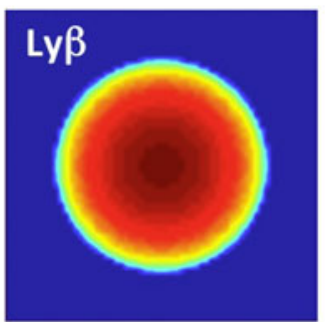

Figure 4. (a) Synthetic MMI data with an appropriate PHA tilt and with the minimum reconstruction width, $w$, much narrower than the spectral linewidth. (b) Ar He- $\beta$ and Ly- $\beta$ images reconstructed from the synthetic MMI data. (c) Expected Ar He- $\beta$ and Ly- $\beta$ images.

images together to approximate narrow-band images, and small discrepancies are unavoidable. The level of discrepancies can be quantified by series of synthetic investigations, which are beyond the scope of the paper and will be discussed elsewhere.

Next, inappropriate MMI data are synthetically created using a uniform sphere of $100 \mu \mathrm{m}$ diameter, $T_{e}=1000 \mathrm{eV}$ and $n_{e}=3 \times 10^{23} \mathrm{~cm}^{-3}$. The PHA pattern and the photonenergy axis are those from the actual inappropriate MMI data shown in Figure 3(a). Thus, the minimum width required to reconstruct the image is $w=20$ pixels (i.e., $44 \mathrm{eV}$ ), as discussed in Section 2. One reason why Figure 3(c) still shows a reasonable image is that $w$ is still narrower than the linewidth $(\sim 60 \mathrm{eV})$, while it is not significantly narrower. 
(a) Synthetic inappropriate MMI data

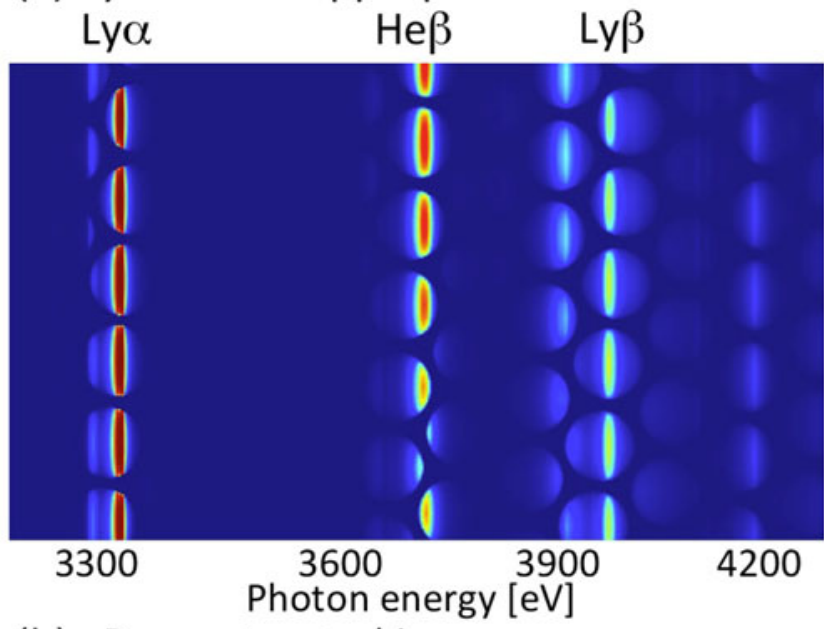

(b) Reconstructed images

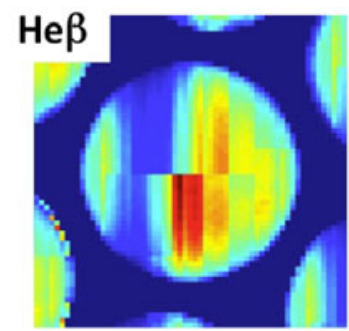

(c) Expected images
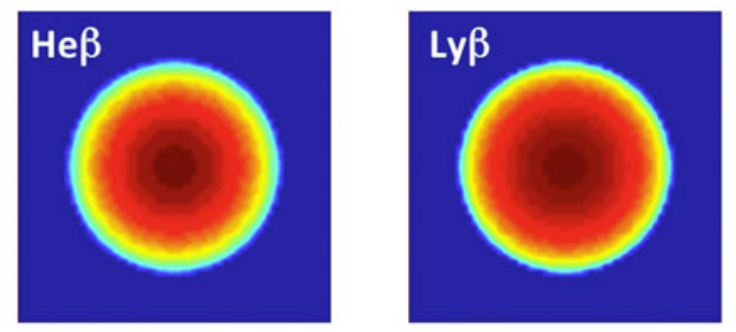

Figure 5. (a) Synthetic MMI data with an inappropriate PHA tilt and with the minimum reconstruction width, $w$, broader than the spectral linewidth. (b) Ar He- $\beta$ and Ly- $\beta$ images reconstructed from the synthetic MMI data. (c) Expected Ar He- $\beta$ and Ly- $\beta$ images.

Thus, to show an extreme case, $n_{e}$ is purposely lowered so that $w$ becomes even broader than its Stark linewidth ( 16-19 eV).

Figure 5(a) displays the resultant synthetic MMI data, which show much narrower spectral features compared with Figure 3(a). Figure 5(b) shows the reconstructed Ar He$\beta$ and Ly- $\beta$ images. Compared with the expected images (Figure 5(c)), the reconstructed images have a lot of artificial structure originating from the discrete nature of the MMI data, which is amplified due to $w>$ FWHM.

\section{Conclusions}

The MMI instrument is an interesting diagnostic tool to study the spatial structure of ICF plasmas. This paper discusses the criterion for reliable MMI diagnostics and its impact on the extracted spatial information. Reliable MMI data have a sampling width, $w$, much narrower than the FWHM of the spectral lines. An appropriate PHA tilt is critical to minimize $w$ for a given target image size and MCP strip width.

By processing synthetically generated MMI data, we demonstrated that MMI data with $w>$ FWHM produce significant bias in the sampled object spatial information. On one hand, the images reconstructed from appropriate MMI data are very similar to those of the expected images. On the other hand, the images from inappropriate MMI data show very different spatial structures from the expected ones. The artificial structure is always vertical due to the fact that the images are reconstructed by superposition of vertical strips originating from different pixel columns. The presence of strong vertical structure in the image is probably a sign that the MMI data are flawed, and is most likely due to not satisfying the criterion. Reliability in the conclusions derived from such inappropriate MMI data is limited.

\section{Acknowledgements}

This work was supported by DOE/NLUF grant nos. DENA0000859 and DE-NA0002267, and LLNL.

\section{References}

1. J. Nuckolls, L. Wood, A. Thiessen, and G. Zimmerman, Nature 239, 139 (1972).

2. J. D. Lindl, P. Amendt, R. L. Berger, S. G. Glendinning, S. H. Glenzer, S. W. Haan, R. L. Kauffman, O. L. Landen, and L. J. Suter, Phys. Plasmas 11, 339 (2004).

3. J. Lindl, O. Landen, J. Edwards, E. Moses, and N. Team, Phys. Plasmas 21, 020501 (2014).

4. D. S. Clark, M. M. Marinak, C. R. Weber, D. C. Eder, S. W. Haan, B. A. Hammel, D. E. Hinkel, O. S. Jones, J. L. Milovich, P. K. Patel, H. F. Robey, J. D. Salmonson, S. M. Sepke, and C. A. Thomas, Phys. Plasmas 22, 022703 (2015).

5. J. A. Koch, T. W. Barbee, N. Izumi, R. Tommasini, R. C. Mancini, L. A. Welser, and F. J. Marshall, Rev. Sci. Instrum. 76, 073708 (2005).

6. R. Tommasini, J. A. Koch, N. Izumi, L. A. Welser, R. C. Mancini, J. Delettrez, S. Regan, and V. Smalyuk, Rev. Sci. Instrum. 77, 10E303 (2006).

7. L. A. Welser, R. C. Mancini, J. A. Koch, S. Dalhed, R. W. Lee, I. E. Golovkin, F. Marshall, J. Delettrez, and L. Klein, Rev. Sci. Instrum. 74, 1951 (2003).

8. N. Izumi, T. W. Barbee, J. A. Koch, R. C. Mancini, and L. A. Welser, Rev. Sci. Instrum. 77, 083504 (2006).

9. T. Nagayama, R. C. Mancini, R. Florido, R. Tommasini, J. A. Koch, J. A. Delettrez, S. P. Regan, and V. A. Smalyuk, J. Appl. Phys. 109, 093303 (2011).

10. T. Nagayama, R. C. Mancini, R. Florido, D. Mayes, R. Tommasini, J. A. Koch, J. A. Delettrez, S. P. Regan, and V. A. Smalyuk, Phys. Plasmas 21, 050702 (2014).

11. T. Nagayama, R. C. Mancini, R. Florido, R. Tommasini, J. A. Koch, J. A. Delettrez, S. P. Regan, V. A. Smalyuk, L. A. Welser-Sherrill, and I. E. Golovkin, Rev. Sci. Instrum. 79, 10E921 (2008)

12. T. Nagayama, R. C. Mancini, R. Florido, D. Mayes, R. Tommasini, J. A. Koch, J. A. Delettrez, S. P. Regan, and V. A. Smalyuk, Phys. Plasmas 19, 082705 (2012). 
13. J. MacFarlane, I. Golovkin, R. Mancini, L. Welser, J. Bailey, J. Koch, T. Mehlhorn, G. Rochau, P. Wang, and P. Woodruff, Phys. Rev. E 72, 066403 (2005).

14. M. F. Gu, Can. J. Phys. 86, 675 (2008).

15. R. Florido, R. Rodríguez, J. M. Gil, J. G. Rubiano, P. Martel, E. Mínguez, and R. C. Mancini, Phys. Rev. E 80, 56402 (2009).
16. J. C. Stewart and K. D. J. Pyatt, Astrophys. J. 144, 1203 (1966).

17. R. C. Mancini, R. F. Joyce, and C. F. Hooper, Jr., J. Phys. B: At. Mol. Phys. 20, 2975 (1987).

18. R. C. Mancini, D. P. Kilcrease, L. A. Woltz, and C. F. Hooper, Jr., Comput. Phys. Commun. 63, 314 (1991).

19. D. Mihalas, Stellar Atmospheres, Astronomy and Astrophysics Series, (W. H. Freeman, 1978). 